\title{
Conductores infractores, ¿Un perfil de conducta desviada?: Análisis de las diferencias y similitudes con una muestra de conductores de la población general.
}

\author{
Beatriz González Iglesias \& José Antonio Gómez Fraguela \\ Universidad de Santiago de Compostela
}

\begin{abstract}
RESUMEN
Con las últimas modificaciones del Código Penal en materia de seguridad vial determinados comportamientos del conductor han adquirido la consideración de delito y pueden ser castigados con penas de prisión. Esta medida ha supuesto un complemento al llamado "permiso de conducción por puntos" y ha propiciado un incremento en la investigación sobre las variables relacionadas con el comportamiento infractor del conductor. En esta línea, el objetivo de este trabajo ha sido comprobar si existen diferencias entre los conductores de la población general y los infractores en una serie de características de personalidad y en el modo en que experimentan y manifiestan sus emociones al volante. Además, también hemos querido analizar sus hábitos de conducción y su historial de multas y accidentes con el fin de determinar si existe un perfil infractor y desviado en los conductores condenados por delitos contra la seguridad vial. Para ello se ha realizado una encuesta a un grupo de conductores condenados por delitos de tráfico y a un grupo de conductores de la población normal. Este último lo componían 647 conductores de la población gallega con edades comprendidas entre los 19 y los 74 años, siendo el $52 \%$ mujeres y el $48 \%$ varones. Los infractores fueron seleccionados de entre aquellos que participaron en un curso de reeducación impartido por psicólogos del COP Galicia. Los resultados muestran que los penados puntúan más alto en Impulsividad que los conductores de la población general y que suelen manifestar su ira al volante mediante conductas de agresión física. Además, los condenados por delitos de tráfico consumen más alcohol, infringen más las normas de circulación y se ven implicados en más accidentes sin víctimas. Estos resultados tienen importantes implicaciones prácticas a la hora de diseñar programas de prevención e intervención en el campo de la seguridad vial.
\end{abstract}

Palabras clave: Delitos de tráfico; impulsividad; ira al volante; alcohol 


\begin{abstract}
Drivers offenders, a profile of deviant behavior? Analysis of differences and similarities with a sample of drivers in the general population. With the latest amendments to the Penal Code in road safety certain driving behaviors have acquired the consideration of crime and may be punishable with imprisonment. This has been a complement to the call "demerit point system" and has led to an increase in research on variables related to the unlawful conduct of the driver. In this line, the aim of this study was to determine whether there are differences between the drivers of the general population and offenders in a number of personality traits and how they experience and express their emotions at the wheel. In addition, we wished to examine their driving habits and history of fines and accidents in order to determine if there is a deviant profile offender and drivers convicted of crimes against road safety. A survey was made with a group of drivers convicted of traffic offenses and a control group. The latter was made up 647 drivers of the Galician population aged 19 to 74 years, and 52\% women and $48 \%$ men. Offenders were selected from among those who participated in a re-education course taught by psychologists from COP Galicia. The results show that offenders scored higher on impulsivity than drivers of the general population and often express their anger by physical aggressive behaviors. In addition, those convicted of traffic offenses consume more alcohol, more violate traffic rules and are involved in more accidents without victims. These results have important implications in the design of prevention and intervention programs in the field of road safety.
\end{abstract}

Keywords: traffic offenses; impulsiveness; driving anger; alcohol

\title{
1. Introducción
}

El llamado "permiso de conducción por puntos", implantado en España en el año 2006 trajo consigo un cambio en el sistema sancionador existente hasta la fecha (Ley 17/2005 por la que se regula el permiso y la licencia de conducción por puntos y se modifica el texto articulado de la ley sobre tráfico, circulación de vehículos a motor y seguridad vial). Además de endurecer las medidas previstas para los conductores infractores, la nueva normativa contempla una serie de programas de sensibilización y reeducación vial orientados a modificar las actitudes de los conductores hacia el cumplimiento de las normas de tráfico y aquellas otras variables que puedan estar relacionadas con su comportamiento infractor al volante (Orden INT/2596/2005, de 28 de julio, por la que se regulan los cursos de sensibilización y reeducación vial para los titulares de un permiso o licencia de conducción). 
Este cambio en el sistema sancionador se ha completado con la entrada en vigor de la Ley Orgánica 15/2007, de 30 de noviembre por la que se modificaba el Código Penal en materia de seguridad vial. Con esta reforma, determinadas conductas como conducir a velocidad excesiva, con tasas de alcoholemia superiores a 1.2 gramos/litro en sangre, de forma manifiestamente temeraria o sin permiso han adquirido la consideración de delito y pueden ser castigadas con penas de prisión.

Nuestro sistema penal también contempla que, en determinados casos, las penas de prisión pueden ser conmutadas por Trabajos en Beneficio de la Comunidad (TBC), tal y como aparece recogido en la Ley Orgánica 10/1995, de 23 de noviembre.

Sin embargo, el escaso número de plazas para la realización de los TBC en función del elevado número de penas impuestas es uno de los factores que, sin duda, dificulta su inmediato cumplimiento. Tal y como recoge la última Memoria de la Fiscalía, en enero de 2009 el número de plazas ofertadas para la realización de los TBC ascendía a un total de 11.404 (3.600 en Cataluña), lo cual se revela como insuficiente para cubrir las necesidades existentes. Se estima que el porcentaje de condenas pendientes ronda ya el $80 \%$ y que más de 50.000 están esperando a ser ejecutadas. Esta situación puede provocar que prescriban un gran número de penas, frustrando así su fin resocializador y originando sentimientos de impunidad.

Pero por otra parte, este nuevo escenario legal ha propiciado, dentro de la tríada vehículo-vía-conductor (Montoro, Alonso, Esteban y Toledo, 2000), un incremento en la investigación sobre las variables relacionadas con el llamado factor humano. Se ha estimado que entre el 70 y el 90 por ciento de los siniestros tienen su causa en el conductor, ya sea por un error involuntario o una infracción deliberada (Pérez, Lucas, Dasi y Quiamzade, 2002). Esto convierte al comportamiento del conductor en el principal objetivo de una buena parte de las intervenciones preventivas y a la Psicología del Tráfico y la Seguridad Vial en una disciplina clave para lograr una reducción significativa de la siniestralidad.

Básicamente, existen dos aspectos del comportamiento del conductor que parecen estar relacionados con la probabilidad de que se vea envuelto en un accidente de tráfico, sus habilidades y destrezas al volante (o la falta de ellas) y su estilo de conducción (habitualmente arriesgado y temerario) (Elander, West y French, 1993). Los déficits de habilidades se traducen en Errores, generalmente involuntarios y no 
intencionados, asociados a fallos en los mecanismos cognitivos y de procesamiento de la información. Las conductas deliberadamente infractoras o Violaciones, que no sólo pueden explicarse recurriendo a argumentos racionales sino también a través de los aspectos de carácter emocional y motivacional (Reason, Manstead, Stradling, Baxter y Campbell, 1990), tienen un mayor impacto sobre la seguridad vial y han acaparado la intención de los investigadores sobre el riesgo y la conducta desviada.

Bajo el auspicio de las teorías de propensión al accidente muchas han sido las variables que se han estudiado como posibles correlatos de las conductas de riesgo al volante (para una revisión, véase Beirness, 1993; Elander, West y French, 1993; Lawton y Parker, 1998) pero la búsqueda de sensaciones, la impulsividad y la hostilidad/ira han ocupado, sin duda, un papel central.

Actualmente el concepto de propensión al accidente (y sus equivalentes) todavía resulta controvertido y sus defensores y detractores se cuentan por igual. Lo que sí parece claro es que los accidentes no se distribuyen de forma aleatoria entre la población y que, tal y como señalan Visser, Pijl, Stolk, Neeleman y Rosmalen (2007), el largo historial de siniestros y repetidos incidentes en la carretera que acumulan algunos individuos no se explica solamente por azar.

Pero, ¿en qué medida los delincuentes de tráfico se diferencian de otro tipo de delincuentes y del resto de conductores? ¿Poseen realmente ciertas características que les hacen más proclives a infringir las normas de circulación? ¿Son personas normales que se transforman cual "Dr. Jekyll" y "Mr. Hyde" cuando se ponen al volante de un automóvil o realmente podemos decir que las personas con ciertas características son más propensas a conducir de forma arriesgada, esto es, que realmente conducen como viven?

Estos interrogantes constituyen el punto de partida de esta investigación en la que hemos querido comprobar si existen diferencias entre los conductores de la población general y los infractores en una serie de características de personalidad y en el modo en que experimentan y manifiestan sus emociones al volante. Además, también hemos querido analizar sus hábitos de conducción y su historial de multas y accidentes con el fin de determinar si existe claramente un perfil infractor y desviado en los conductores condenados por delitos contra la seguridad vial. 


\section{Método}

\subsection{Participantes}

Participaron en este estudio 702 conductores procedentes de dos muestras, una extraída de la población general y la otra compuesta por conductores infractores. La primera la componían 647 conductores con edades comprendidas entre los 19 y los 74 años $($ Media $=38.88$; D.t. $=11.95)$, siendo el 52\% mujeres y el $48 \%$ varones.

La muestra de penados por delitos contra la seguridad vial estaba compuesta por 55 conductores, de los cuales el $90.7 \%$ eran varones. Su media de edad era de 38.84 años, con un rango que oscila entre $\operatorname{los} 18$ y los 73 años.

\subsection{Procedimiento}

Los datos del grupo control fueron recogidos en los centros de reconocimiento de conductores de Galicia colaboradores con la investigación entre aquellas personas que acudían a realizar la revisión psicotécnica para la renovación del carné de conducir. A todos los participantes se les solicitó la colaboración voluntaria en la investigación, garantizándoles en todo momento el anonimato y la confidencialidad de sus datos

Los infractores fueron seleccionados de entre aquellos que participaron en el curso de reeducación en sustitución de los TBC impartido por los psicólogos del Colegio Oficial de Psicología de Galicia en las principales ciudades de la comunidad entre los años 2009 y 2010.

\subsection{Variables e instrumentos}

En este estudio hemos empleado la versión reducida del Cuestionario de Impulsividad de Eysenck $\left(\mathrm{I}_{7}\right)$ desarrollada por Aluja y Blanch (2007). Consta de 24 ítems agrupados en tres subescalas: Impulsividad, Afán de aventuras y Empatía con un formato de respuesta Sí / No. El valor del alpha de Cronbach obtenido en nuestro estudio para cada una de estas subescalas fue de $.73, .79$ y .68 , respectivamente.

Para evaluar o determinar aquellas situaciones que generan ira en la conducción, así como la propensión de los individuos a enfadarse mientras conducen, se empleó una versión reducida del Driving Anger Scale (DAS; Deffenbacher, Oetting y Lynch, 1994) elaborada en un estudio previo (González-Iglesias, 2008). Esta versión del DAS consta de 22 ítems agrupados en seis subescalas: Descortesías, Obstrucciones de tráfico, 
Gestos hostiles, Conducción lenta, Presencia policial y Conducción ilegal. Las distintas subescalas del DAS presentan una consistencia interna satisfactoria, con valores alpha de $.71, .70, .83, .74, .77$ y .79 respectivamente.

Para responder a este cuestionario los sujetos deben imaginar las situaciones descritas en cada uno de los ítems e indicar a continuación el nivel de ira o enfado que les provocan en una escala tipo Likert de 5 puntos $(1=$ Ninguno; $5=$ Muchísimo $)$.

La forma de expresar la ira al volante fue evaluada utilizando también una versión reducida del Driving Anger Expression Inventory (DAX; Deffenbacher, Lynch, Oetting y Swaim, 2002) de elaboración propia. Consta de 18 ítems agrupados en cuatro subescalas (Expresión Verbal, Expresión Física, Uso del vehículo para expresar la ira y Expresión Adaptativa) que los sujetos deben contestar en una escala tipo Likert de 5 puntos $(1=$ Ninguno; 5 = Mucho $)$ en función de la frecuencia de su respuesta colérica al volante.

En nuestro estudio la consistencia interna de las escalas es en todos los casos superior a .70 y sólo la subescala de Uso del vehículo presenta un valor del alpha de Cronbach inferior.

Para evaluar la conducta y las infracciones cometidas por los conductores al volante empleamos la subescala de Violaciones de las normas del Driver Behaviour Questionnaire (DBQ; Reason et al., 1990) adaptada al español por Gras, Sullman, Cunill, Planes, Aymerich y Font-Mayolas (2006). Consta de 9 ítems que los sujetos deben responder en una escala tipo Likert de 5 puntos $(0=$ Nunca; $4=$ Muchas veces $)$ en función de la frecuencia con que han realizado esas conductas en el último año. En nuestro estudio esta subescala presenta una adecuada consistencia interna, con un valor del coeficiente de alpha de Cronbach de .74.

Para evaluar la frecuencia de consumo de alcohol y otras drogas introdujimos una serie de preguntas en las que se pedía a los conductores que indicasen con qué periodicidad solían consumir esas sustancias en una escala de 6 puntos $(0=$ Nunca; $6=$ Casi todos los días). Además, incluimos una serie de ítems relacionados con la frecuencia de consumo y el abuso de alcohol en el último año. Para el registro de estas respuestas se empleó una escala tipo Likert de 5 puntos $(0=$ Nunca; $4=$ Más de 6 veces). 
Adicionalmente solicitamos a los participantes información sociodemográfica y relacionada con su historia como conductores: experiencia (año en que habían obtenido el carné de conducir), exposición (media de kilómetros recorridos al año), número de multas (velocidad, alcohol, uso del móvil, etc.) y accidentes (con y sin víctimas) en los últimos 5 años.

\subsection{Análisis de datos}

En primer lugar realizamos un análisis de correlaciones entre todas las variables incluidas en el estudio. A continuación, llevamos a cabo distintos análisis de varianza para comprobar si existían diferencias entre los conductores penados por delitos de tráfico y los procedentes de la población general en distintas variables psicológicas y relacionadas con la conducción así como en el consumo de alcohol y otras drogas.

Para realizar el análisis de los datos se ha utilizado el paquete estadístico SPSS 15.1 para Windows.

\section{Resultados}

\subsection{Relaciones entre variables}

En la Tabla 1 se incluyen las correlaciones halladas entre las distintas variables evaluadas en este estudio para la muestra global de conductores.

La variable Violaciones de las normas del DBQ se relaciona significativamente con todas las variables psicológicas incluidas en este estudio, con un rango que oscila entre $r=.13$ y $r=.57$.

Los accidentes con víctimas correlacionan negativamente con la faceta de Empatía del $\mathrm{I}_{7}$ y con la variable de Expresión adaptativa de la ira. En sentido positivo se relacionan con la subescala de Uso del vehículo del DAX.

Los accidentes sin víctimas se relacionan en sentido positivo con las facetas de Impulsividad y Afán de aventuras, con las subescalas de Gestos hostiles y Presencia policial del DAS, con las subescalas de expresión desadaptativa de la ira del DAX y con los ítems referidos al consumo y abuso del alcohol. En sentido negativo, los accidentes sin víctimas también correlacionan con la subescala de Empatía del $\mathrm{I}_{7}$. 


\section{Tabla 1}

Correlaciones entre las distintas variables evaluadas en este estudio

\begin{tabular}{|c|c|c|c|c|c|c|c|c|c|}
\hline & \multirow[b]{2}{*}{ 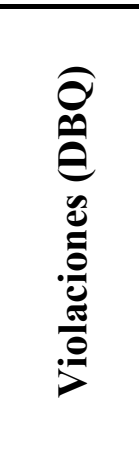 } & \multirow[b]{2}{*}{ 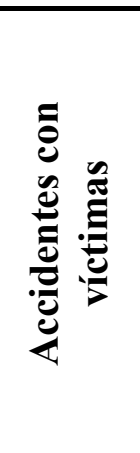 } & \multirow[b]{2}{*}{ 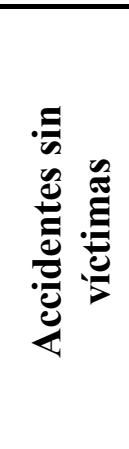 } & \multirow[b]{2}{*}{ 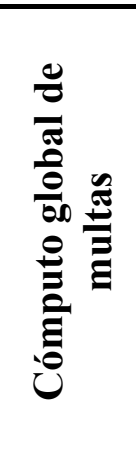 } & \multicolumn{5}{|c|}{ Multas } \\
\hline & & & & & 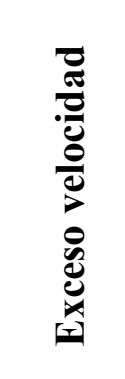 & $\frac{\bar{e}}{\frac{e}{0}}$ & 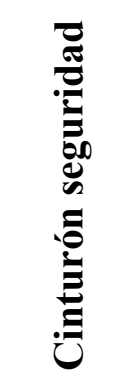 & 疍 & 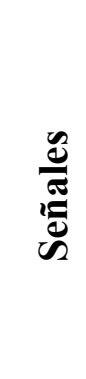 \\
\hline Impulsividad & $.24 * *$ & .01 & $.08^{*}$ & $.13^{* *}$ & $.08^{*}$ & $.09^{*}$ & .06 & .07 & .05 \\
\hline Afán de aventuras & $.38 * *$ & .05 & $.13 * *$ & $.20 * *$ & $.18^{* *}$ & $.13 * *$ & $.08^{*}$ & .03 & .06 \\
\hline Empatía & $-.25 * *$ & $-.18 * *$ & $-.12 * *$ & $-.16^{* *}$ & $-.12 * *$ & -.03 & $-.16 * *$ & $-.10^{*}$ & -.01 \\
\hline Descortesías & $.13 * *$ & -.02 & .03 & -.01 & -.04 & -.01 & .00 & .04 & .00 \\
\hline $\begin{array}{l}\text { Obstrucciones } \\
\text { tráfico }\end{array}$ & $.28 * *$ & -.07 & -.03 & .03 & .00 & -.05 & .07 & .04 & .03 \\
\hline Gestos hostiles & $.24 * *$ & .00 & $.09 *$ & .01 & -.06 & .01 & .06 & .04 & .01 \\
\hline Conducción lenta & $.40 * *$ & .03 & .05 & $.12 * *$ & $.09^{*}$ & -.01 & $.10^{*}$ & $.08^{*}$ & .06 \\
\hline Presencia policial & $.34 * *$ & .08 & $.14^{* *}$ & $.22 * *$ & $.14^{* *}$ & $.11 * *$ & $.18^{* *}$ & $.12 * *$ & $.10^{*}$ \\
\hline Conducción ilegal & $-.12 * *$ & -.03 & -.01 & $-.08^{*}$ & -.05 & $.10 *$ & .00 & -.03 & -.04 \\
\hline Expresión verbal & $.34 * *$ & .06 & $.10 *$ & $.08^{*}$ & $.08^{*}$ & .02 & .00 & .02 & .03 \\
\hline Expresión física & $.39 * *$ & .01 & $.08^{*}$ & $.08^{*}$ & .06 & .05 & .04 & .02 & .04 \\
\hline Uso del vehículo & $.57 * *$ & $.09 *$ & $.12 * *$ & $.17 * *$ & $.12 * *$ & .07 & $.11 * *$ & $.11 * *$ & .05 \\
\hline $\begin{array}{l}\text { Expresión } \\
\text { adaptativa }\end{array}$ & $-.22 * *$ & $-.09 *$ & .02 & -.01 & .00 & .07 & -.03 & $-.08 *$ & .01 \\
\hline Consumo & $.37 * *$ & .00 & $.09 *$ & $.19 * *$ & $.08^{*}$ & $.27 * *$ & $.10 * *$ & $.09^{*}$ & .00 \\
\hline Abuso & $.40 * *$ & .01 & $.11^{* *}$ & $.23^{* *}$ & $.12 * *$ & $.35^{* *}$ & $.10 * *$ & .05 & .04 \\
\hline
\end{tabular}

Revista Española de Investigación Criminológica

Artículo 6, Número 8 (2010) www.criminología.net

ISSN: $1696-9219$ 
El cómputo global de multas se relaciona positivamente con las subescalas de Impulsividad y Afán de aventuras del $\mathrm{I}_{7}$, con las de Conducción lenta y Presencia policial del DAS y con las de Expresión física, Expresión verbal y Uso del vehículo del DAX. Asimismo, el consumo y el abuso del alcohol correlacionan positivamente con las multas. Por su parte, la faceta de Empatía correlaciona de forma negativa y significativa con las multas autoinformadas de los últimos 5 años.

Analizando de forma individual cada una de las multas observamos que las impuestas por exceder el límite de velocidad, por no llevar abrochado el cinturón de seguridad y hablar por el móvil correlacionan positiva y significativamente con las subescalas de Conducción lenta y Presencia policial del DAS, con la variable Uso del vehículo del DAX y con la frecuencia de consumo de alcohol. En sentido negativo, estas tres categorías de multas también se relacionan con la variable Empatía del $\mathrm{I}_{7}$.

Las multas relacionadas con la velocidad excesiva, además, se relacionan positivamente con las facetas de Impulsividad y Afán de aventuras, con la subescala de Expresión verbal del DAX y con el abuso del alcohol. Las sanciones impuestas por alcoholemia positiva presentan correlaciones positivas y significativas con las subescalas de Impulsividad y Afán de aventuras del $\mathrm{I}_{7}$, con las de Presencia policial y Conducción ilegal del DAS y con las dos medidas empleadas en este estudio para evaluar la frecuencia de consumo y abuso del alcohol.

Además de las ya señaladas, las multas por no llevar abrochado el cinturón de seguridad se relacionan en sentido positivo con la faceta de Afán de aventuras del $\mathrm{I}_{7}$. Por su parte, las sanciones impuestas a los conductores por el uso del móvil al volante correlacionan positivamente con la frecuencia de consumo de alcohol y negativamente con la variable Expresión adaptativa del DAX. Saltarse un semáforo en rojo o no respetar las señales de STOP se relaciona únicamente con el enfado suscitado por los conductores ante la presencia de la policía vigilando el tráfico.

\subsection{Diferencias entre los penados y los conductores de la población general}

Los penados por delitos de tráfico puntúan más alto que los conductores de la población general en las subescalas de Afán de aventuras y Impulsividad del $\mathrm{I}_{7}$, pero sólo las diferencias halladas en Impulsividad son estadísticamente significativas $(F=$ $4.37, p<.05)$. 
A grandes rasgos observamos también que los conductores de la población general muestran más enfado que los penados por delitos de tráfico ante las distintas situaciones de la conducción evaluadas mediante el DAS. En la Figura 1 aparecen representadas las puntuaciones de los conductores en aquellos ítems del DAS en que se han observado diferencias significativas entre ambos grupos. El exceso de velocidad y la presencia de un vehículo que zigzaguea entre los demás coches para abrirse paso son los ejemplos de Conducción ilegal que más enfadan a los conductores de la población general. Estos conductores, además, se muestran más molestos que los penados ante algunas Descortesías de los demás conductores (Un vehículo da marcha atrás delante de usted sin mirar), las muestras de hostilidad (Un conductor toca el claxon como protesta por su forma de conducir) y las situaciones de tráfico lento.

Por su parte, la presencia de la policía vigilando el tráfico es la única situación que genera más enfado a los condenados por delitos contra la seguridad vial que a los conductores de la población general $(F=4.26, p<.05)$.

\section{Figura 1}

Diferencias entre las puntuaciones de los penados y los conductores de la población general en los items del DAS

Un conductor circula más despacio de lo que es razonable

Un conductor circula muy por encima del límite de velocidad

Un policía le hace detener su vehículo

Un conductor está a parcando muy lentamente e interrumpe el tráfico

Un vechículo va adelantando a los coches por la derecha y por la izquierda

Un conductor toca el claxon como protesta por su forma de conducir

Un vehículo da marcha a trás delante de usted sin mirar
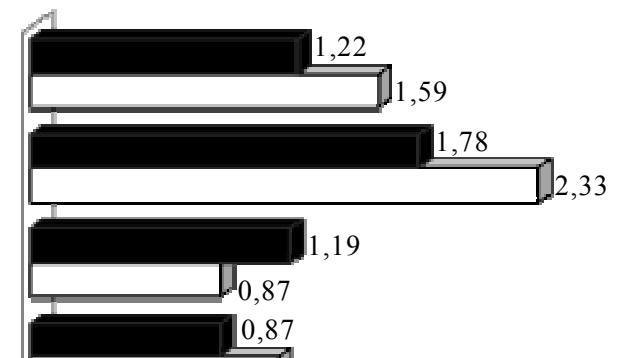

1,14

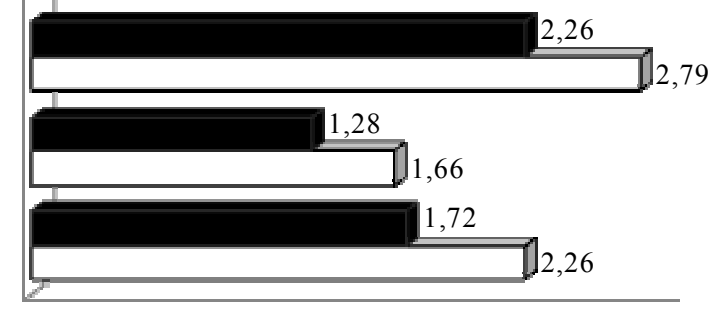

1

2

Penados $\square$ Población general 
Los datos recogidos mediante el DAX y representados en la Figura 2 nos indican que el empleo de gestos obscenos es estadísticamente más frecuente entre los penados que entre los conductores de la población general $(F=3.83, p<.05)$, quienes suelen manifestar su ira mediante expresiones de tipo verbal o para-verbal (i.e., comentarios negativos o movimientos de reproche con la cabeza).

\section{Figura 2}

Diferencias entre las puntuaciones de los penados y los conductores de la población general en los items del DAX

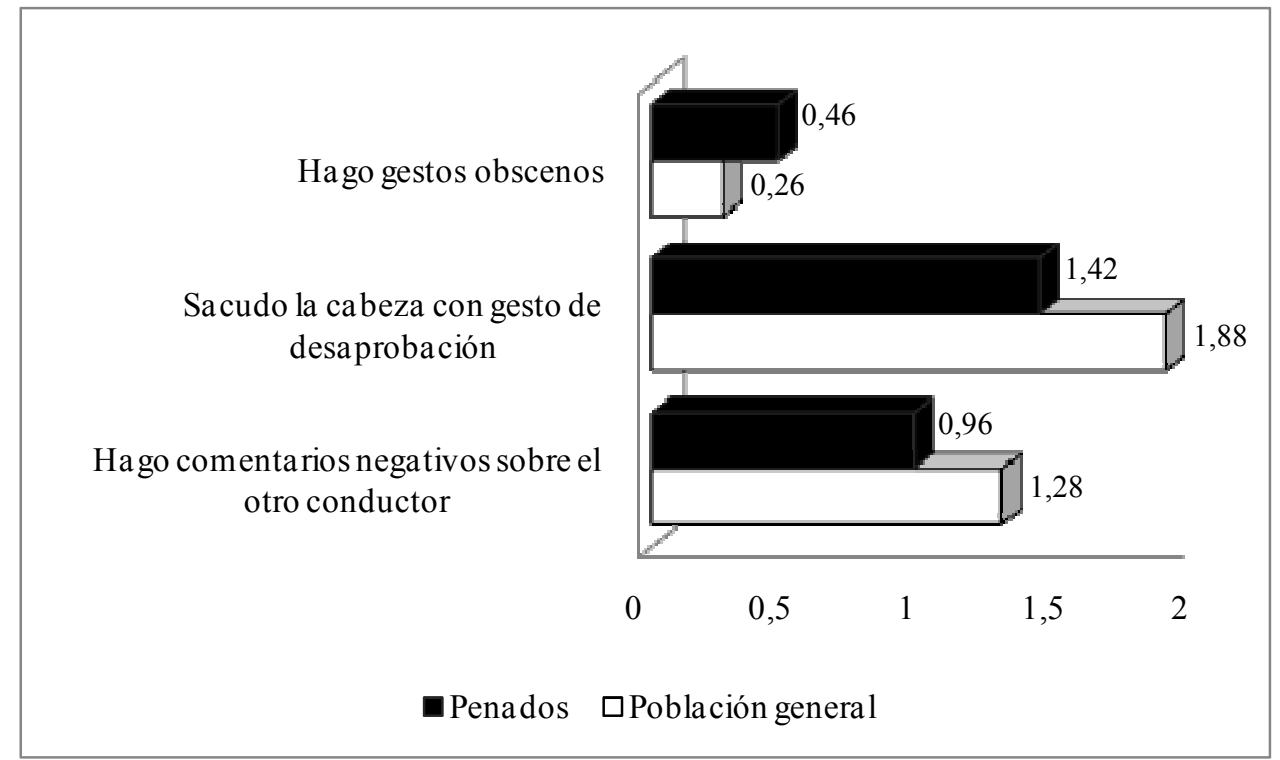

También hemos encontrado diferencias entre ambos grupos en la subescala de Violaciones de las normas del DBQ $(F=8.27, p<.01)$, donde los condenados por delitos de tráfico puntúan significativamente más alto que los conductores del grupo control ( $M s=.55$ y .49). Analizando de forma individual los ítems que componen esta escala observamos que el exceso de velocidad en carretera y la conducción bajo los efectos del alcohol son las conductas aberrantes más frecuentemente informadas por los penados (véase Figura 3). 


\section{Figura 3}

Diferencias entre las puntuaciones de los penados y los conductores de la población general en los items de la subescala de Violaciones de las normas del DBQ

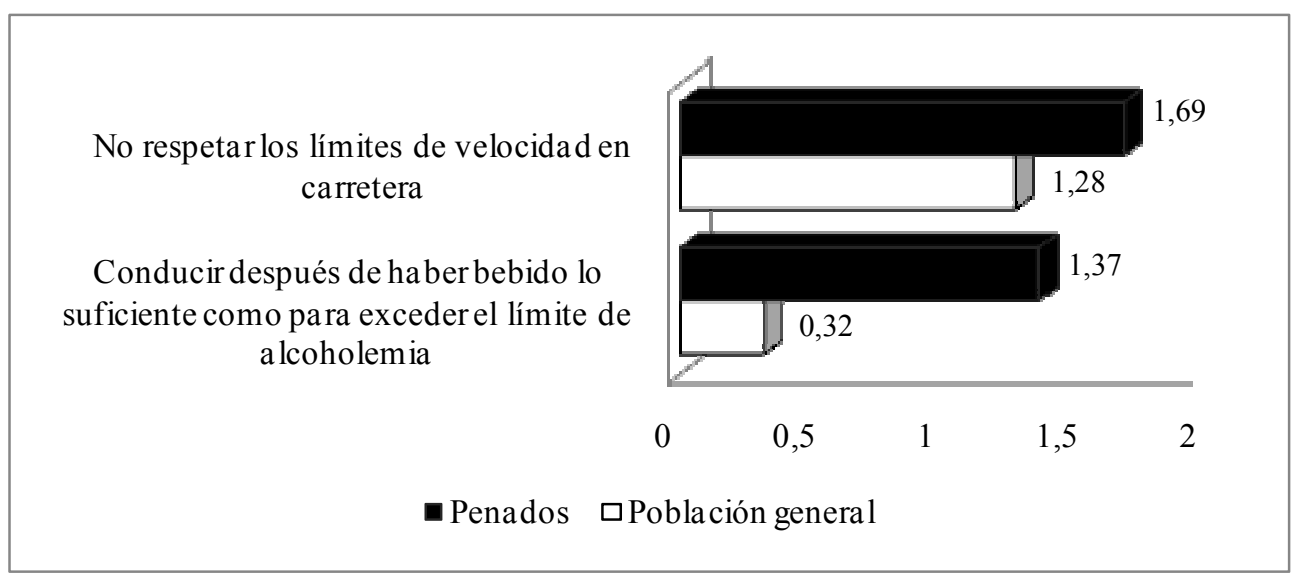

Los resultados anteriores coinciden con los datos procedentes de las multas que los participantes en nuestro estudio informan que han recibido en los últimos años. De nuevo, son los penados los que puntúan más alto en el cómputo global de multas $(M s=$ 2.57 y $.88, F=65.88, p<.001)$ e informan de más sanciones por exceso de velocidad y alcoholemia positiva, siendo esta última diferencia especialmente significativa (véase Figura 4).

\section{Figura 4}

Diferencias entre las multas autoinformadas por los penados y los conductores de la población general

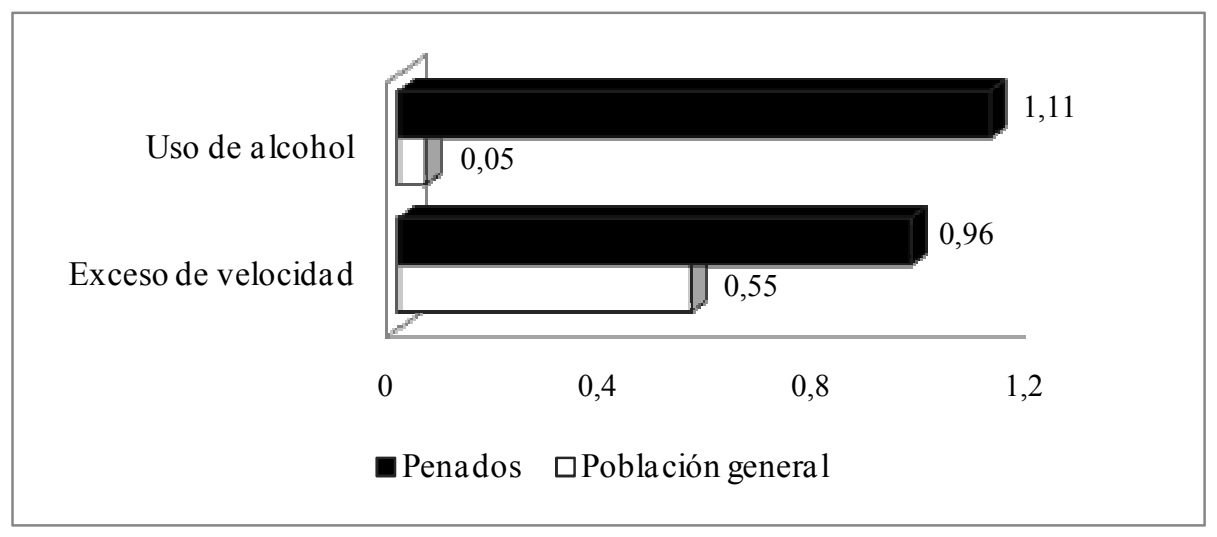


$\mathrm{Y}$ aunque no se registran diferencias entre ambos grupos en los accidentes con víctimas, los penados reconocen que han tenido más accidentes sin víctimas en los últimos 5 años $(M S=1.44$ y .99$)$, siendo esta diferencia estadísticamente significativa $(F=8.26, p<.01)$.

También hemos hallado diferencias significativas en la frecuencia de consumo de alcohol y otras sustancias. Tal y como aparece recogido en la Figura 5, los condenados por delitos de tráfico informan de un mayor consumo de alcohol (vino, cerveza y licores) y drogas (cannabis y cocaína) que los conductores de la población general.

\section{Figura 5}

Diferencias en el consumo de alcohol y otras drogas de los penados y los conductores de la población general

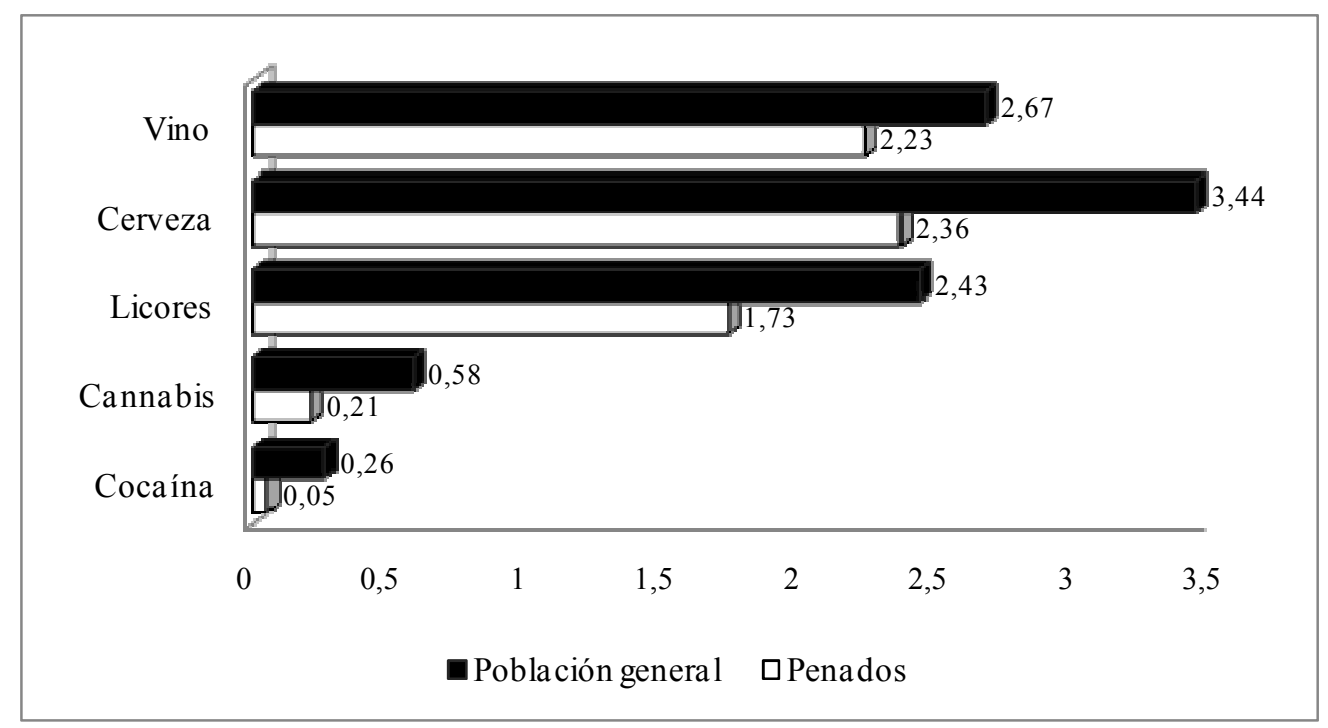

Además, los penados consumen más alcohol del que tenían previsto inicialmente $(M=2.04)$ con mayor frecuencia que los conductores del grupo control $(M=.96)$, siendo esta diferencia estadísticamente significativa $(F=36.69, p<.001)$. Asimismo, hemos hallado diferencias significativas en el consumo abusivo de alcohol o de riesgo, siendo los penados por delitos de tráfico los conductores que con más frecuencia suelen ingerir seis o más bebidas alcohólicas en una misma ocasión $(M s=1.94$ y $.67, F=$ 52.22, $p<.001)$. 


\section{Discusión}

Los resultados de nuestro estudio ponen de manifiesto la importancia de las variables del conductor en la explicación de su conducta infractora al volante. En concreto, hemos comprobado cómo un estado emocional iracundo y ciertas características de personalidad -básicamente, Impulsividad y Afán de aventuras- se relacionan con un comportamiento más antinormativo del conductor (i.e., consumo de alcohol al volante, exceso de velocidad, incumplimiento de las normas de tráfico, etc.) y una mayor implicación en accidentes de tráfico. Estos resultados coinciden con los hallados en otros estudios donde también se ha destacado el papel de estas variables en la predicción de la conducta infractora y arriesgada al volante (Gómez-Fraguela y González-Iglesias, 2010; Dahlen, Martin, Ragan y Kuhlman, 2005; Deffenbacher, Deffenbacher, Lynch y Richards, 2003; Owsley, McGwin y McNeal, 2003; Schwebel, Severson, Ball y Rizzo, 2006).

Asimismo, nuestros resultados confirman la existencia de diferencias entre los conductores de la población general y los condenados por delitos contra la seguridad vial. En general, los penados infringen más las normas de tráfico y por ello reciben más multas (sobre todo por exceso de velocidad y consumo de alcohol al volante) y se ven involucrados en más accidentes de tráfico "menores" o sin víctimas mortales.

También se han observado diferencias significativas entre ambos grupos en el modo de expresar su ira cuando conducen. Los penados por delitos contra la seguridad vial suelen expresar su enfado mediante conductas de agresión física (i.e., gestos obscenos); por su parte, los conductores del grupo control suelen emplear con más frecuencia expresiones verbales y señales paralingüísticas de carácter agresivo. Y dado que el enfado y las reacciones agresivas del conductor son un buen predictor de la conducta infractora al volante (Dahlen et al., 2005; Furnham y Saipe, 1993; Lajunen, Parker y Stradling, 1998; Delhome y Villieux, 2005), sería interesante que los programas de reeducación vial incluyesen algún módulo destinado al (auto)control emocional y al manejo de la ira.

Además, los penados puntúan más alto en Impulsividad que los conductores de la población general, especialmente en aquellos ítems referidos a la impulsividad de tipo 
cognitivo (i.e., hacer y decir cosas sin pensar). Se ha sugerido que las medidas reeducadoras más convencionales que apelan únicamente a la razón y a la toma de conciencia mediante argumentos racionales pueden no ser persuasivas para aquellos conductores que suelen actuar de forma espontánea e irreflexiva (Ledesma, Poó y Peltzer, 2007; Ulleberg, 2002). En estos casos, el diseño de intervenciones y mensajes efectivos pasa por tener en cuenta las características de estos conductores y centrarse en los aspectos motivacionales y emocionales que regulan la conducta infractora.

Los resultados de nuestro estudio también nos indican que los penados presentan un mayor consumo de alcohol y otras sustancias (básicamente cannabis y cocaína). Este resultado va en la línea de lo hallado en los estudios con internos condenados por delitos contra la seguridad vial de las cárceles catalanas (Herraiz, 2009; Monras, Aparicio, López y Pons, 2009), en los que se ha puesto de manifiesto que los problemas relacionados con el alcohol constituyen una de las características más sobresalientes del perfil psicológico de los infractores de tráfico.

Este hallazgo es especialmente relevante de cara a la prevención y la intervención ya que diversos estudios han constatado que el consumo (y el abuso) de alcohol se hallan estrechamente relacionados con la conducta infractora al volante (Dobson, Brown, Ball, Powers y McFadden, 1999, Donovan, Marlatt y Salzberg, 1983; Wilson y Jonah, 1985). Además, el consumo de alcohol al volante es una práctica habitual en nuestro país (Del Río, González-Luque y Álvarez, 2001) y se calcula que entre el 30 y el 50\% de los accidentes mortales están relacionados con el alcohol, especialmente durante la noche y el fin de semana (Calafat, Adrover, Juan y Blay, 2008).

Por ello, resulta fundamental que los programas de tratamiento de infractores incluyan también algún componente centrado en reestructurar las distorsiones cognitivas de los conductores y en desmontar los falsos mitos y las creencias erróneas que éstos manejan sobre los efectos del alcohol y sobre las acciones que pueden realizar para neutralizar sus efectos antes de ponerse al volante (e.g., beber café, tomar aire fresco o una ducha de agua fría, etc.).

Desde el punto de vista metodológico nuestros resultados confirman la validez de los autoinformes en la recogida de datos sobre los conductores. El 90\% de los penados que participaban en el curso de reeducación impartido por el COP Galicia 
había sido condenado por exceder el límite de velocidad o sobrepasar los límites legales de alcoholemia permitidos, un dato que se ha confirmado con las respuestas obtenidas por los propios conductores en los distintos cuestionarios empleados para evaluar su conducta infractora al volante y su nivel de consumo de drogas y alcohol.

Con todo, es importante tener en cuenta que la muestra de penados que hemos empleado en esta investigación acudía a los cursos de reeducación de forma totalmente voluntaria y que, por lo tanto, es probable que conformen un grupo de conductores más normativo y socializado que el que pudiera hallarse, por ejemplo, en un centro penitenciario.

Por ello, sería conveniente que, en la medida en que se vayan cumpliendo las condenas pendientes de ser ejecutadas, se fuesen introduciendo en el estudio grupos de condenados por delitos contra la seguridad vial más heterogéneos o que estén cumpliendo su pena en distintos contextos con el fin de poder analizar no sólo sus diferencias con respecto a los conductores de la población general sino también los distintos perfiles de conductores infractores. 


\section{Referencias}

Aluja, A. y Blanch, A. (2007). Comparison of impulsiveness, venturesomeness and empathy (I7) structure in English and Spanish samples: Analysis of different structural equation models. Personality and Individual Differences, 43, 22942305.

Beirness, D. (1993). Do we really drive as we live? The role of personality factors in road crashes. Alcohol, Drugs, and Driving, 9, 129-143.

Calafat, A., Adrover, D., Juan, M. y Blay, N. T. (2008). Relación del consumo de alcohol y drogas de los jóvenes españoles con la siniestralidad vial durante la vida recreativa nocturna en tres comunidades autónomas en 2007. Revista Española de Salud Pública, 82, 323-331.

Dahlen, E. R., Martin, R. C., Ragan, K. y Kuhlman, M. M. (2005). Driving anger, sensation seeking, impulsiveness, and boredom proneness in the prediction of unsafe driving. Accident Analysis and Prevention, 37, 341-348.

Deffenbacher, J. L., Deffenbacher, D. M., Lynch, R. S. y Richards, T. L. (2003). Anger, aggression, and risky behaviour: A comparison of high and low anger drivers. Behaviour Research and Therapy, 41, 701- 718.

Deffenbacher, J. L., Lynch, R. S., Oetting, E. R. y Swaim, R. C. (2002). The Driving Anger Expression Inventory: A measure of how people express their anger on the road. Behaviour Research and Therapy, 40, 717-737.

Deffenbacher, J. L., Oetting, E. R. y Lynch, R. S. (1994). Development of a Driving Anger Scale. Psychological Reports, 74, 83-91.

Delhomme, P. y Villieux, A. (2005). Adaptation française de l'échelle de colère au volant D.A.S.: Quels liens entre colère éprouvée au volant, infractions et accidents de la route déclarés par de jeunes automobilistes? / French adaptation of the driving anger scale (D.A.S.): Which links between driving anger, violations and road accidents reported by young drivers? European Review of Applied Psychology / Revue européenne de psychologie appliqué, 55, 187-205.

Dobson, A., Brown, W., Ball, J., Powers, J. y McFadden, M. (1999). Women drivers' behaviour, socio-demographic characteristics and accidents. Accident Analysis and Prevention, 31, 525-535.

Donovan, D. M., Marlatt, G. A. y Slazberg, P. M. (1983). Drinking behavior, personality factors and high-risk driving. A review and theoretical formulation. Journal of Studies on Alcohol, 44, 395-428.

Elander, J., West, R. y French, D. (1993). Behavioral correlates of individual differences in road-traffic crash risk: An examination of methods and findings. Psychological Bulletin, 113, 279-294.

Fiscalía General del Estado (2009, 20 de octubre). Memoria 09 del Fiscal de Sala de Seguridad Vial. Recuperado el 19 de febrero de 2010, de http://www.fiscal.es/cs/Satellite?cid=1240559967757\&language=es\&pagename $=$ PFiscal $\% 2 F P a g e \% 2 F F G E \_$buscadorDocEspecialista .

Furnham, A. y Saipe, J. (1993). Personality correlates of convicted drivers. Personality and Individual Differences, 14, 329-336.

Gómez-Fraguela, J. A. y González-Iglesias, B. (2010). El papel de la personalidad y la ira en la explicación de las conductas de riesgo al volante en mujeres jóvenes. Anales de Psicología, 26(2), 318-324. 
González-Iglesias (2008). Road rage: el papel de la ira en la conducción. Memoria de licenciatura. Santiago de Compostela.

Gras, M. E., Sullman, M. J. M., Cunill, M., Planes, M., Aymerich, M. y Font-Mayolas, S. (2006). Spanish drivers and their aberrant driving behaviours. Transportation Research: Part F, 9, 129-137.

Herraiz, F. (2009, Diciembre). Descripción del perfil psicológico de los internos por delitos contra la seguridad del tráfico. Invesbreu, 48, 9-11.

Lajunen, T., Parker, D. y Stradling, S. G. (1998). Dimensions of driver anger, aggressive and highway code violations and their mediation by safety orientation in UK drivers. Transportation Research Part F: Traffic Psychology and Behaviour, 1, 107-121Lawton, R. y Parker, D. (1998). Individual differences in accident liability: A review and integrative approach. Human Factors, 40, 655671.

Ledesma, R, Poó, F. y Peltzer, R. (2007). Búsqueda impulsiva de sensaciones y comportamiento de riesgo en la conducción. Avaliaçao Psicológica, 6, 117-125.

Ley Orgánica 10/1995, de 23 de noviembre, del Código Penal. BOE n ${ }^{\circ}$ 281, 24 de noviembre de 1995.

Ley 17/2005, de 19 de julio, por la que se regula el permiso y la licencia de conducción por puntos y se modifica el texto articulado de la ley sobre tráfico, circulación de vehículos a motor y seguridad vial. BOE nº 172, 20 de julio del 2005.

Ley Orgánica 15/2007, de 30 de noviembre, por la que se modifica la Ley Orgánica 10/1995, de 23 de noviembre, del Código Penal en materia de seguridad vial. BOE $\mathrm{n}^{\circ} 288,1$ de diciembre del 2007.

Monras, M., Aparicio, A., López, J. A. y Pons, I. (2009, Diciembre). Estudio de las variables presentes en los penados por delitos de conducción bajo los efectos del alcohol (contra la seguridad vial). Invesbreu, 48, 6-9.

Montoro, L., Alonso, F., Esteban, C. y Toledo, F. (2000). Manual de seguridad vial: el factor humano. Barcelona: Ariel-INTRAS.

Orden INT/2596/2005, de 28 de julio, por la que se regulan los cursos de sensibilización y reeducación vial para los titulares de un permiso o licencia de conducción. BOE $n^{\circ} 190,10$ de agosto del 2005.

Owsley, C., McGwin, G. y McNeal, S. F. (2003). Impact of impulsiveness, venturesomeness, and empathy on driving by older adults. Journal of Safety Research, 34, 353-359.

Pérez, J. A., Lucas, A., Dasi, F. y Quiamzade, A. (2002). La desobediencia masiva al código de circulación. Normas heterónomas frente a las normas interindividuales. Psicothema, 14, 788-794.

Reason, J. T., Manstead, A., Stradling, S. y Baxter, J. S. (1990). Errors and violations on the roads: A real distinction? Ergonomics, 33, 1315-1332.

Schwebel, D. C., Severson, J., Ball, K. K. y Rizzo, M. (2006). Individual difference factors in risky driving: The roles of anger/hostility, conscientiousness, and sensation-seeking. Accident Analysis and Prevention, 38, 801-810.

Ulleberg, P. (2002). Personality subtypes of young drivers. Relationship to risk-taking preferences, accident involvement, and response to a traffic safety campaign. Transportation Research Part F: Traffic Psychology and Behaviour, 4, 279-297.

Wilson, R. J. y Jonah, B. A. (1985). Identifying impaired drivers among the general driving population. Journal of Studies on Alcohol, 46, 531-537. 


\section{Correspondencia con la autora:}

Beatriz González Iglesias

Universidad de Santiago de Compostela

Facultad de Psicología - Departamento de Psicología Clínica

Campus Vida, s/n

15782 - Santiago de Compostela

Email: beatriz.gonzalez.iglesias@usc.es 